\title{
LAS FUNCIONES DE POPULARIDAD*. Estado de la cuestión y principales debates
}

\author{
Agustí Bosch \\ Universitat Autònoma de Barcelona \\ E-mail: agusti.bosch@uab.es
}

Aida Díaz

Universitat Autònoma de Barcelona

Clara Riba

Universitat Pompeu Fabra

\begin{abstract}
RESUMEN
El artículo pasa revista a las investigaciones sobre la relación que existe entre la evolución de la intención de voro a los gobiernos y la coyuntura económica. Se constata que los ciudadanos suelen premiar con su voto a los gobiernos que mandan durante épocas de prosperidad, y viceversa. El artículo desmenuza la modelización que han adquirido estas funciones: qué tipos de variables han englobado, qué retardos temporales se han utilizado y qué valor explicativo han conseguido. Finalmente se entra en la discusión de los principales debates que las funciones de popularidad han generado: ¿influye más sobre el voto la evolución de las condiciones económicas personales del votante o de las condiciones macroeconómicas del país?; ¿̇influyen más las evaluaciones del pasado o las expectativas sobre el futuro? En otro artículo inminente, los autores presentarán los resultados de una función de popularidad para el gobierno español entre 1984 y 1996.
\end{abstract}

\section{INTRODUCCIÓN}

Las funciones de popularidad de los gobiernos aparecen como resultado del interés general por conocer las relaciones entre economía y política. Ya

* Este artículo es el primer fruto del proyecto de investigación La evolución del apoyo popular a los gobiernos: economía, politicas y coaliciones financiado por la CICYT (ayuda núm. SEC 960510). Otros miembros del equipo son Josep M. VAll. FS y Joan FoNT (UAB). En un artículo inmimente "Funciones de popularidad (y 2): el caso español» se presentarán los resultados de la función estimada para el caso del gobierno español entre 1984 y 1996.

\section{Reis}


desde principios de siglo se puso de manifiesto el deseo de entender cómo la situación económica de un país influye en el nivel de aceptación que tiene su gobierno entre la ciudadanía. Así, en los años treinta, empezamos a encontrar algunos estudios que se centraron en el análisis del efecto que la prosperidad o la depresión económica podían tener en las posibilidades de reelección de los candidatos (ver Kramer, 1971, para un resumen). Sin embargo, hay que esperar a la década de los setenta, cuando se publican los tres trabajos seminales de Mueller (1970), Goodhart y Bhansali (1970) y Kramer (1971), para que la literatura sobre el voto económico adquiera relevancia. Es a partir de estos artículos cuando un número creciente de investigadores se interesa por el tema y cuando se consolidan grupos de académicos interesados en modelar la influencia de la economía en la politica. Actualmente, además de mantenerse el interés por el tema, se ha ampliado el número de países estudiados, de tal manera que las funciones de popularidad constituyen hoy una rama específica de la literatura sobre comportamiento electoral (ver Nannestad y Paldam, 1994, para una síntesis de la literatura).

En realidad, se estiman dos tipos de funciones diferentes: las funciones de voto y las funciones de popularidad. La diferencia entre ellas estriba únicamente en el indicador que se utiliza para medir el grado de aceptación del gobierno. En principio, el mejor indicador del respaldo de los ciudadanos a la gestión del gobierno es el porcentaje de votos que obtiene el partido que lo sustenta en las siguientes elecciones generales. Éste es el indicador que utilizan las funciones de voto. Sin embargo, su principal problema estriba en su escasez. Las elecciones generales se realizan cada cierto período, habitualmente cuatro años, con lo cual los investigadores están obligados a considerar largos períodos de tiempo con el fin de tener suficientes datos para realizar el análisis. En el caso de democracias jóvenes como es la española, esto representa un claro inconveniente.

Un indicador alternativo del apoyo gubernamental entre la ciudadanía es el que se obtiene de encuestas periódicas de opinión. En ellas se pregunta a los encuestados por su opinión sobre la gestión del gobierno. Éste es el indicador que utilizan las funciones de popularidad. Su principal ventaja estriba en su frecuencia. La abundancia de observaciones permite estimar los efectos independientes de las distintas variables económicas al posibilitar la aplicación de controles estadísticos.

A pesar de sus evidentes ventajas sobre las funciones de voto, las funciones de popularidad también presentan ciertos inconvenientes. En primer lugar, el indicador utilizado se obtiene a partir de una muestra $y$, por consiguiente, contiene un error de muestreo, cosa que no ocurre con el porcentaje de voto, que se obtiene a partir de todos los electores. En segundo lugar, no tenemos certeza sobre la fiabilidad que se puede otorgar a las respuestas dadas por los encuestados. Es importante recordar aquí el problema de las no respuestas y el problema de las respuestas no sinceras en los datos de encuesta. En cambio, el voto es la expresión explícita de la voluntad de los electores y, por lo tanto, los resultados electorales son el respaldo real que tiene el partido del gobierno. 
En los modelos que estamos describiendo podemos realizar una segunda distinción según el nivel de análisis utilizado. Es evidente que los análisis que utilizan funciones de voto son siempre agregados debido a la propia naturaleza de los resultados electorales. Sin embargo, en los modelos que usan funciones de popularidad caben las dos opciones. En la primera, los investigadores pueden decidir agregar las respuestas individuales y realizar un análisis agregado. En este caso, los análisis agregados con funciones de popularidad hacen hincapié, al igual que las funciones de voto, en las relaciones entre condiciones macroeconómicas y comportamiento colectivo. En la segunda opción, los investigadores pueden realizar un análisis a nivel individual, en cuyo caso es posible contrastar hipótesis sobre las motivaciones individuales de los electores y proporcionar la conexión necesaria entre comportamiento individual y colectivo de los ciudadanos.

También hay que destacar que existe un importante grupo de investigadores que también trabajan en el estudio de las relaciones entre economía y política pero asumiendo una causalidad inversa a la que se supone en las funciones de popularidad. Es decir, este grupo está interesado en explicar las políticas económicas a partir de fenómenos políticos, y no a la inversa. Así, mediante las llamadas funciones de reacción, modelan los efectos de la popularidad de los gobiernos en los instrumentos de política económica que los mismos implementan. Se supone que los gobiernos quieren ser reelegidos y que conocen el efecto que una mejora de determinados indicadores económicos tiene sobre su propia popularidad. A partir de estas hipótesis, los gobiernos pueden manipular los instrumentos de política económica que tienen a su disposición, tales como gasto público, transferencias corrientes, políticas de empleo o políticas fiscales, a fin de mejorar sus posibilidades electorales en aquellas situaciones en que padecen un déficit de popularidad. Este segundo aspecto, sin embargo, ha recibido una mayor atención entre los economistas que entre los politólogos (ver, por ejemplo, Golden y Poterba, 1980).

\section{Bases teóricas de las funciones de popularidad}

Las bases teóricas de las funciones de voto y popularidad se deben fundamentalmente a Schumpeter (1945) y Downs (1957). Schumpeter, en su obra Capitalism, Socialism and Democracy, propone una nueva teoría de la democracia en la que considera que, además de las públicas, existen motivaciones privadas en política. A diferencia de la teoría clásica que considera las elecciones de representantes como un medio para conseguir el objetivo primordial de dar el poder de decisión al pueblo, la democracia de Schumpeter, invirtiendo el orden de estos dos elementos, es considerada como un sistema institucional en el que los individuos adquieren el poder de decidir mediante una lucha competitiva por el voto de los ciudadanos. Esta noción de competencia política por el poder que introduce Schumpeter es la idea clave que desarrolla posterior- 
mente Downs en su obra An Economic Theory of Democracy (1957). En ella, Downs no sólo considera que los políticos tienen intereses privados (altruistas o no), sino que considera también que los electores son racionales, en el sentido de que son capaces de comparar las distintas alternativas que se le ofrecen y de votar por el partido o candidato que esperan que les proporcione una mayor urilidad.

Es precisamente en esta visión de la democracia en la que se hallan las bases de la llamada hipótesis de la responsabilidad. Esta hipótesis sostiene que los ciudadanos responsabilizan al gobierno de la situación económica por la que atraviesa el país. Es decir, consideran que, en un momento dado, los indicadores que reflejan el estado de la macroeconomía son el resultado de las decisiones tomadas anteriormente por los políticos y altos cargos del gobierno. En consecuencia, si la situación económica es buena, premian al gobierno con su voto; pero si la situación económica es mala, lo castigan negándole su apoyo y votando a uno de los partidos de la oposición. En definitiva, las elecciones se tratarían de una especie de plebiscito sobre la actuación del gobierno, lo cual - por otra parte- es una concepción bastante extendida en Ciencia Política. Hay que destacar que existe una clara asimetría en el conocimiento que los electores tienen acerca de la previsible actuación de las distintas fuerzas políticas. Mientras que tienen buenas pistas sobre la previsible actuación del partido en el gobierno y, también, alguna idea sobre la de otros partidos que puedan haber tenido responsabilidades de gobierno anteriormente, no tienen ninguna indicación sobre el comportamiento esperado de los partidos que han estado siempre en la oposición. No obstante, según la hipótesis de la responsabilidad, ello no es ningún obstáculo para decidir su voto, puesto que los ciudadanos tienen en este aspecto un comportamiento conservador. Asumen que, si el estado de la economía es razonablemente bueno, la gestión del gobierno es aceptable $y$, por lo tanto, le mantienen su confianza volviendo a votar por el partido que lo sostiene. En cambio, si el estado de la economía es realmente malo, suponen que la gestión del gobierno es mejorable, retirándole por lo tanto su confianza y votando a alguno de los partidos de la oposición, dándole así a éste la oportunidad de cambiar el estado de las cosas.

La hipótesis de la responsabilidad es ampliamente aceptada por la comunidad científica debido tanto a la plausibilidad del comportamiento que asume como al hecho de haber sido contrastada con resultados positivos en casi todos los estudios realizados con funciones de popularidad. No obstante, existe también una segunda hipótesis sobre los fundamentos de las funciones de popularidad. Se trata del llamado policy-oriented voting. En vez de escoger entre votar o no votar al partido del gobierno, esta hipótesis considera que los electores escogen a qué partido votar en función de la capacidad que tiene cada uno de ellos para solucionar los temas problemáticos. Como se supone que los partidos de izquierdas están más preocupados por el desempleo y menos por la inflación que los de derechas, los ciudadanos votarán mayoritariamente a un partido de izquierdas cuando la tasa de paro es alta y a un partido de derechas 
cuando sube la tasa de inflación. Esta segunda hipótesis también ha sido contrastada positivamente en unos pocos estudios y goza de mucho menor consenso entre los investigadores que la primera (ver Kieweit, 1981, y Rattinger, 1991).

\section{Breve historia de las funciones de popularidad}

Podemos considerar que la literatura sobre funciones de popularidad se inicia en 1970 con la aparición de los tres trabajos seminales citados anteriormente. Los estudios previos que habían tratado la relación entre economía y política eran técnicamente muy sencillos, consistiendo básicamente en cálculos de porcentajes y en análisis de correlaciones. Sus autores estaban más interesados en establecer la existencia de la relación que en cuantificarla. Sin embargo, a partir de 1970 , los investigadores empiezan a realizar análisis multivariantes, aplicando técnicas econométricas y controlando por distintas variables a fin de separar los efectos independientes. Los pioneros en esta nueva etapa fueron los ya citados Mueller (1970) y Kramer (1971), en los Estados Unidos, y Goodhart y Bhansali (1970), en el Reino Unido. El artículo de Mueller, que trabaja con una función de popularidad del Presidente de los Estados Unidos, es endeble desde el punto de vista estadístico, pero es muy rico conceptualmente. Introduce por primera vez algunas variables políticas que son clásicas de las funciones de popularidad y está repleto de ideas sugestivas que han sido recogidas y desarrolladas por otros investigadores en trabajos posteriores. En cambio, el trabajo de Kramer, que estima una función de voto para las elecciones al Congreso desde 1896 hasta 1964, destaca por sus logros metodológicos, por su calidad estadística y por su elegancia. El tercer estudio, el de Goodhart y Bhansali, consiste en un análisis de las series de popularidad de partidos y líderes políticos entre 1947 y 1968 . A pesar de carecer de la intuición del de Mueller y del rigor estadístico del de Kramer, este trabajo destaca por su exhaustividad y completitud.

Estos tres análisis tuvieron la virtud de abrir un debate general entre los politólogos. Se escribieron muchos artículos debatiendo la metodología y técnicas aplicadas y discutiendo los resultados a los que habían llegado. Se replicaron los modelos de voto y popularidad y se extendieron a otros países, como Alemania, Francia, Japón y los países nórdicos. A finales de los setenta se produjo un cambio cualitativo en los modelos que se aplicaban. Por un lado, en Zurich, un grupo importante de investigadores empezó a relacionar las funciones de popularidad con las funciones de reacción, combinándolas en un único modelo político-económico. Se trataba de un modelo cerrado donde casi todas las variables eran endógenas. Frey y Schneider (1979) son los autores del modelo más completo de este grupo, cuyos resultados se caracterizan por un excelente ajuste estadístico. Por otro lado, y en paralelo a los trabajos de grupo de Zurich, apareció en Harvard otro grupo de trabajo alrededor de las funciones de popularidad, liderado por Hibbs, que desarrolló un nuevo modelo. 
Hibbs (1982) considera que cada gobierno, al inicio de su mandato, dispone de un estoque de popularidad que va depreciándose a lo largo del tiempo, pero que puede aumentar o disminuir en función de si los resultados económicos son buenos o malos. La importancia de los trabajos de Hibbs se debe tanto a su novedoso enfoque como a la introducción de algunas características técnicas importantes, tanto por lo que se refiere a modelos no lineales como a su estimación por nuevos métodos.

Durante la década del ochenta y a principios de la del noventa, los investigadores han estado envueltos en una importante discusión acerca de los motivos individuales del comportamiento electoral. A pesar del consenso generado por la teoría del voto retrospectivo de Fiorina (1981), según la cual los ciudadanos utilizan su experiencia pasada para formar sus expectativas de futuro, no existe unanimidad sobre qué aspectos del pasado son los que realmente cuentan en el momento de decidir el voto: la situación económica personal o la situación económica general del país (véase más adelante el epígrafe Sociotrópico o egotrópico). El artículo de Kinder y Kieweit (1981), defendiendo que lo que influye en los electores es la situación económica general, causó un gran impacto y generó una oleada de réplicas. La crítica más importante que recibió fue la de Kramer (1983), basada en aspectos metodológicos y técnicos. Orros politólogos entraron en la polémica, pero después de replicar el análisis de Kinder y Kieweit, la mayoría llegaron a la misma conclusión. Uno de los trabajos más importantes en este sentido es el estudio de Lewis-Beck (1988) comparando las funciones de popularidad de cinco países europeos con las de Estados Unidos.

Actualmente, las líneas de investigación sobre funciones de popularidad se han diversificado. En primer lugar, algunos estudios se han centrado en analizar si la función de popularidad es la misma para electores de distintos grupos socioeconómicos. Por ejemplo, Jonung y Wadensjö (1987) encuentran distintas funciones de popularidad para distintos grupos de edad, nivel de renta y sexo. En segundo lugar, otros estudios se han centrado en descubrir si las funciones de popularidad varían o no entre distintas regiones de un mismo país. Así, Rattinger (1991) ha estimado para el caso alemán funciones de popularidad por distritos, introduciendo como nuevas variables explicativas las características estructurales de cada zona. En resumen, podríamos decir que durante los últimos años estamos asistiendo a un intento de generalización de las funciones de popularidad, con estudios que las aplican a distintos países, a distintos grupos y a distintas situaciones.

\section{LOS MODELOS}

Los modelos de voto y popularidad se acostumbran a especificar como modelos de regresión en los que la variable dependiente se estima como función de tres grupos de variables. El primer grupo es el de las variables de tipo 
económico; el segundo, el de las variables de tipo politico; y el tercero, el de las variables que podríamos llamar de estructura del modelo, es decir, ciertas variables que son necesarias para especificarlo correctamente pero que no pueden incluirse en ninguna de las dos categorías precedentes. Sin embargo, debe destacarse que el tratamiento dado a las distintas componentes del modelo no es homogéneo, siendo su componente económica mucho más elaborada que su componente política. Este hecho puede parecer narural dado que, inicialmente, estos modelos fueron planteados para estudiar el efecto de las variables económicas en el voto y sólo más tarde, cuando se vio la necesidad de controlar por los efectos de determinados sucesos políticos, empezaron a usarse variables explicativas de tipo político. Sin embargo, el dominio de la parte económica sobre la política en estos modelos se debe principalmente a la facilidad para la obtención de gran cantidad de indicadores macroeconómicos fiables, que contrasta con la dificultad de operacionalizar fenómenos políticos y la inexistencia de instituciones que elaboren y distribuyan regularmente indicadores políticos. Además, y como consecuencia del tipo de variables disponibles, continuas las económicas y generalmente categóricas las políticas, el análisis de la parte económica de los modelos es mucho más sofisticado que el de la parte política

\section{La componente económica}

Llamamos componente económica a la parte del modelo que contiene el conjunto de variables que reflejan el estado de la economía. Entre ellas podemos distinguir las que son variables objetivas de las que son subjetivas. Las variables objetivas son indicadores macroeconómicos del país, generalmente procedentes de estadísticas oficiales, entre las que cabe destacar la tasa de paro y la tasa de inflación. En prácticamente todos los estudios, estas variables resultan ser significativas y tienen un impacto negativo sobre la popularidad: un aumento de la tasa de paro o un aumento de los precios provocan una pérdida de popularidad del gobierno. Sin embargo, algunos autores (Hibbs, 1982) han puntualizado que el efecto de la inflación no es lineal: una inflación baja no afecta al gobierno, pero cuando ésta supera el 10 por 100 su efecto sobre la popularidad es muy importante. Además de las dos citadas, existe otro grupo de variables macroeconómicas que son significativas en algunos modelos pero cuyo comportamiento no es tan sistemático como el de las dos anteriores. Entre ellas podemos destacar la renta real disponible (ver ejemplos en Kramer, 1971; Tufte, 1978; Frey y Schneider, 1978, y Golden y Poterba, 1980) y el Producto Interior Bruto (ver un ejemplo en Tufte, 1978), que son variables a menudo significativas y siempre con efectos positivos; el tipo de cambio, que con frecuencia es significativo pero cuyo efecto no es claro, puesto que el coeficiente estimado es negativo en algunos estudios pero positivo en otros (por ejemplo, Sanders, Ward y Marsh, 1987, estiman un coeficiente negativo para el Reino Unido, mientras que Mancha, 1993, lo estima positivo para el mode- 
lo español). Finalmente, el efecto negativo de la presión fiscal ha sido demostrado en varios estudios, algunos de cuyos autores mantienen que, al igual que ocurre con la inflación, su efecto no es lineal, siendo sólo un factor explicativo clave cuando supera ciertos límites. En definitiva, se puede afirmar que, entre las variables objetivas macroeconómicas, la tasa de paro y la inflación son las únicas que tienen un efecto claro y estable (negativo) en la popularidad del gobierno, mientras que el comportamiento de las demás es más bien irregular.

Por su parte, las variables subjetivas son las que reflejan las percepciones que los ciudadanos tienen sobre la situación económica. Se obtienen de las respuestas a preguntas específicas sobre temas económicos que regularmente se incluyen en las encuestas de opinión. El indicador de percepción generalmente se construye como la diferencia entre el porcentaje de gente que tiene una opinión favorable sobre el estado de la economía y el porcentaje de gente que la tiene desfavorable. Las preguntas casi siempre tienen una perspectiva temporal, estando redactadas en términos de una comparación entre la situación actual y la anterior o bien entre la situación actual y la situación previsible futura. Ello nos permite distinguir entre variables subjetivas retrospectivas y variables subjetivas prospectivas según que el momento respecto del que se compara la situación actual sea pasado o futuro. Por otra parte, podemos clasificar también estas variables según el objeto al que hacen referencia las preguntas. Éstas pueden referirse a la propia situación económica del individuo o bien a su visión de la situación económica general del país en su conjunto. En el primer caso, la persona responde teniendo en cuenta únicamente su situación personal o la de sus allegados, mientras que en el segundo responde únicamente teniendo en cuenta su percepción sobre la economía colectiva, con independencia de su propia situación.

\section{La componente politica}

Entre las variables políticas hay dos que pueden considerarse clásicas y que fueron introducidas a principios de los setenta por Mueller (1970) y Kramer (1971), respectivamente. La primera es la llamada rally around the flag, que podríamos traducir por reacción patriótica. Esta variable intenta recoger el efecto que tiene la política exterior del gobierno en su popularidad. Cualquier suceso político internacional en el que el país está involucrado y que tiene amplia repercusión en los medios de comunicación se considera un momento crítico. Por ejemplo, intervenciones militares, cumbres diplomáticas, negociaciones políticas o similares. Se supone que, en estas situaciones, los ciudadanos tienen predisposición a defender el papel de su país y, si el gobierno maneja favorablemente la crisis, se espera que se produzca un aumento de su populari$\mathrm{dad}$. Sin embargo, se asume que este incremento es temporal y que la popularidad irá disminuyendo paulatinamente hasta el siguiente momento crítico. El valor que toma esta variable en cada instante es igual al período de tiempo 
transcurrido desde el último punto crítico. En consecuencia, se espera que tenga un coeficiente negativo puesto que su valor aumenta con la distancia al punto crítico, mientras que la popularidad disminuye.

La segunda de las variables políticas clásicas es la llamada coattail effect o efecto de arrastre. Cuando se convocan dos elecciones simultáneamente, como, por ejemplo, presidenciales y legislativas o bien generales y regionales, se supone que las elecciones de segundo orden quedan contaminadas por las elecciones de primer orden. Es decir, que durante la campaña tienen una mayor repercusión las propuestas de los partidos y los candidatos de la elección considerada más importante, de tal manera que parte de los electores vota en las elecciones de segundo orden en clave de las de primer orden. Este efecto de arrastre parece que se da incluso entre elecciones cercanas en el tiempo aunque no sean simultáneas. A pesar de que existen algunas especificaciones más sofisticadas, la manera más sencilla de modelar este efecto es introducir como variable explicativa el porcentaje de votos obtenido por el mismo partido en las elecciones de mayor rango y se espera que su coeficiente sea positivo.

Las demás variables políticas acostumbran a operacionalizarse como variables dicotómicas que toman el valor uno cuando se produce el suceso y cero en los demás casos. Son variables definidas con el objeto de modelar fenómenos políticos impredecibles que aparecen en un determinado momento, como escándalos de corrupción, crisis de gobierno o acciones militares. Por ejemplo, este tipo de variables han sido usadas para recoger los efectos de un caso de corrupción fiscal y de desavenencias entre gobierno y presidente de la República en Francia (Hibbs, 1981); para el escándalo Watergate en Estados Unidos (Chappell y Keech, 1985); para ver el efecto de la formación de una coalición entre dos partidos y la incidencia de la guerra de las Malvinas en el Reino Unido (Clarke, Steward y Zuk, 1986; Norporh, 1987; Clarke, Misler y Whiteley, 1990; Sanders, Ward y Marsh, 1987), o para determinar el efecto de una crisis política en España (Amor Bravo, 1985).

En alguna ocasión ha sido posible definir también algunas variables políticas ordinales que, sin ser de tipo continuo, suministran una información más fina que las dicotómicas. En concreto, para modelar el efecto de la guerra del Vietnam se ha utilizado como indicador el número de soldados americanos muertos en la contienda durante cada período, cosa que permite no tan sólo detectar el efecto de la existencia de la guerra, sino también el de sus incidencias (Chappell y Keech, 1985).

\section{La componente de estructura del modelo}

Esta parte del modelo contiene la constante de la ecuación más un conjunto de variables que, a pesar de que aparecen como resultado de la actividad política, no son estrictamente políticas en el sentido que no reflejan sucesos políticos específicos. Así, la constante de la ecuación expresa el nivel de acepta- 
ción que tiene el gobierno a largo plazo. Se puede interpretar como el voto básico del partido que lo sostiene, es decir, aquel que proviene de los electores con una clara identificación con el partido gobernante. En algunas ocasiones se utilizan variables dicotómicas para distinguir los períodos correspondientes a cada jefe de gobierno. Estas variables valen uno durante el mandato de un determinado presidente y cero en los demás períodos. Los coeficientes estimados para cada mandato permiten medir los efectos de las personalidades de los presidentes en la popularidad de sus gobiernos (para el caso español, ver Amor Bravo, 1985).

Para modelar las tendencias a largo plazo, las funciones de popularidad generalmente incluyen, además de la constante, otras tres variables. La primera es la llamada tasa de depreciación, introducida por Hibbs y Vasilatos (1981). Expresa qué parte de la popularidad del período precedente todavía permanece en el período actual. Si el coeficiente estimado tiene valores altos, cercanos a uno, debe interpretarse que el pasado influencia en gran medida la evaluación del presente, mientras que si tiene valores bajos significa que los efectos de la popularidad pasada desaparecen rápidamente.

La segunda variable es el cost of ruling o desgaste del gobierno, que intenta recoger la lenta y constante pérdida de popularidad que sufre cualquier gobierno a lo largo de la legislatura debido a su propia actividad. Fue introducida por Mueller (1970) argumentando que, puesto que ningún gobierno puede cumplir todas las expectativas generadas durante la campaña electoral, sus decisiones inevitablemente decepcionan a una parte de su electorado que, en consecuencia, le retira su apoyo. El indicador que habitualmente se utiliza para esta variable es el número de períodos de tiempo transcurridos desde el inicio del mandato y se espera que el signo del coeficiente estimado sea negativo.

La tercera de las variables de tendencias a largo plazo es la que modela la creciente responsabilidad del gobierno sobre el estado de la economía a lo largo de su mandato. Se supone que, cuando un partido gana las elecciones y llega al gobierno, hereda una situación económica derivada de la actuación del gobierno anterior. Sin embargo, a medida que transcurre el tiempo y va tomando decisiones de gobierno, cada vez es más responsable del estado de la economía, hasta serlo totalmente. Esta responsabilidad creciente se estima multiplicando las variables económicas por la raíz cuadrada del tiempo transcurrido desde el acceso al poder del gobierno. Así, durante los primeros años, el efecto de las variables económicas es pequeño pero aumenta rápidamente, mientras que, más tarde, es grande pero con aumentos lentos (Chappell y Keech, 1985).

Finalmente, las funciones de popularidad también acostumbran a tener variables que modelan efectos sistemáticos a corto plazo. En concreto, modelan los ciclos electorales que consisten en aumentos y disminuciones regulares de popularidad entre dos elecciones consecutivas (Goodhart y Bhansali, 1970; Nordhaus, 1975). Un ciclo electoral consta de tres fases: una euforia postelectoral llamada luna de miel, que dura alrededor de cuatro meses; una segunda 
fase en la que se produce un descenso de la popularidad; y un nuevo aumento de popularidad en los seis meses anteriores a la siguiente elección, que ayuda al partido del gobierno a volver a ganar las elecciones. Para recoger el efecto de este ciclo se utiliza una función cuadrática del tiempo transcurrido desde la anterior elección.

\section{Principales resultados}

Por lo que se refiere a su formulación, cabe destacar la falta de unanimidad entre los investigadores sobre cuál es la mejor especificación y el mejor método de estimación de las funciones de popularidad. Los estudios realizados son longitudinales, transversales o una combinación de ambos; algunos son modelos estáticos mientras que otros son dinámicos; algunos consideran que lo que es relevante es el valor de las variables económicas mientras que otros consideran que lo que es importante son sus variaciones; habitualmente se utilizan funciones lineales, pero algunos autores han demostrado la no linealidad de ciertas relaciones. Sin embargo, a pesar de estas discrepancias de planteamiento, hay un acuerdo general en los resultados: existe un efecto estadísticamente significativo de las variables que reflejan el estado de la economía en el voto, de tal manera que, si la situación económica es buena, las probabilidades de reelección del partido del gobierno aumentan y, si es mala, las que aumentan son las probabilidades de los partidos de oposición. En la mayoría de los estudios se confirma, pues, la validez de la hipótesis de la responsabilidad para gobiernos estables.

Entre las variables macroeconómicas, las dos que tienen un efecto más consistente en la popularidad son la tasa de paro y la inflación. Ambas tienen unos coeficientes estimados que oscilan entre $-0,4$ y $-0,8$ en los países desarrollados (Nannestad y Paldam, 1994). Es decir, un aumento de un punto en la tasa de paro o en la inflación se espera que produzca una disminución de entre 0,4 y 0,8 puntos en el porcentaje de popularidad o de voro. Referente a las demás variables, ninguna de ellas tiene un efecto definido, existiendo una cierta inestabilidad tanto de su valor como incluso de su signo.

Finalmente, sea cual sea la especificación del modelo, se puede afirmar que el ajuste de las funciones de popularidad es en general muy elevado. El porcentaje de variación explicada oscila entre el 75 y el 95 por 100 en casi todos los estudios, a pesar de que sólo un tercio de este porcentaje puede atribuirse a las variables económicas. Además, probablemente debido a que se estiman con datos de encuesta, el valor del coeficiente de determinación $\mathrm{R}^{2}$ es habirualmente más alto en las funciones de popularidad que en las de voto. La necesidad de contestar rápidamente a una pregunta inesperada y la ausencia de consecuencias de sus manifestaciones induce a los encuestados a dar respuestas más directas y menos reflexivas que los votos que depositan en unas elecciones. Por lo tanto, los datos de encuesta reflejan mejor las reacciones de los ciudadanos a 
las fluctuaciones económicas que los votos y, en consecuencia, la parte económica del modelo explica mejor las variaciones en la popularidad que los resultados electorales (Inoguchi, 1980).

Actualmente, los autores están modelizando una función de popularidad para el gobierno del PSOE entre 1983 y 1995. Aunque presentar sus resultados no sea el objetivo de este artículo, el siguiente párrafo presenta algunos de ellos a modo de primicia (tómense, pues, todas las prevenciones habituales para estos casos).

Según cálculos provisionales, parece ser que el paro fue la principal magnitud macroeconómica que dañó la popularidad de los gobiernos González. En concreto, cada punto de la tasa de aceleración del paro generó una caída de dos puntos y medio de popularidad, y cada punto de la tasa de aceleración de la inflación generó una caída de popularidad de dos tercios de punto. Debe subrayarse que no fue la tasa de paro lo que incidió sobre esa popularidad, ni tan sólo su tasa de crecimiento, sino la tasa de aceleración de ese crecimiento. También deben subrayarse las demoras temporales con que actuaron estas variables: de tres trimestres en el caso del paro y de dos trimestres en el caso de la inflación. Otra curiosidad es que parece que el impacto del paro fue decreciendo a lo largo de los años hasta hacerse casi imperceptible al final del período.

Otra variable que tuvo un fuerte impacto sobre la popularidad del gobierno fue la expectativa de mejora de la economía general. Por cada punto de crecimiento de esas expectativas, la popularidad creció en 0,16 puntos con una demora de un trimestre. También hubo un claro impacto del liderazgo — si bien mucho menos significativo que el de las variables anteriores-. Por cada punto de valoración que González arrancaba a los sucesivos líderes del PP, mejoraba la popularidad del gobierno en casi tres puntos. Es destacable que este impacto es independiente de los anteriores y su inclusión en el modelo no afecta a su solidez.

La tasa de depreciación media de todo el período es del 0,95 - muy alta si se compara con la de países de nuestro entorno-. No obstante, esta tasa esconde dos períodos muy distintos: hasta 1993 la tasa de depreciación está a niveles totalmente normales $(0,7-0,8)$, pero a partir de 1993 se eleva casi a la unidad indicando que se instaura una fuerte inercia de la popularidad (o más bien impopularidad) del gobierno. La conjetura más plausible es que ello fuera debido a la fuerte acción de ciertos medios de comunicación.

Finalmente, existen tres momentos atípicos que se deben modelizar como variables dicotómicas. Son el momento de la firma del Tratado de adhesión a la CE, la invasión de Kuwait y la explosión del escándalo Filesa. Este último hecho le costó inicialmente más de cuatro puntos de popularidad, pero si se agregan otros cuatro del siguiente trimestre menos la acción de la tasa de depreciación, le llegó a costar más de ocho puntos. Debido a la alta tasa de depreciación de ese período, siete trimestres después de la aparición del escándalo, todavía habían más de seis puntos de castigo al PSOE que se debían imputar a ese hecho. 
Ésos son primeros resultados que deberán refinarse y estudiar mucho más, pero que aparecerán próximamente en la literatura académica.

\section{LOS DEBATES SUSTANTIVOS}

Una vez expuestos tanto las bases teóricas de las funciones de popularidad (sección 1) como el tipo de modelos que estas funciones suelen producir (sección 2), esta sección se concentrará en discutir las principales controversias que estas funciones han engendrado entre la familia académica.

\section{¿Economia objetiva o percepciones subjetivas?}

En la sección 2 hemos mostrado que la componente económica de una función de popularidad puede contener tanto condiciones macroeconómicas objetivas como percepciones subjetivas acerca de esas condiciones económicas. Por tanto, una cuestión importante es si la popularidad gubernamental es consecuencia de la primera o de la segunda.

Los resultados empíricos sugieren que las percepciones son mucho más importantes que la economía objetiva. Dos de los pioneros (Hibbs, 1979, y Fiorina, 1978 y 1981) ya debían entrever algo de este tipo cuando introdujeron - respectivamente- datos sobre las preocupaciones económicas de la gente y diversas reflexiones sobre la presentación de las condiciones económicas por parte de los partidos políticos. Pero la verdadera pugna para promocionar las percepciones económicas al reino de la variable decisiva llegó con Sanders et al. (1987), quienes revelan su impacto sobre la popularidad gubernamental por encima de las variables objetivas.

Una función de popularidad construida a partir de percepciones de la economía en vez de hechos macroeconómicos es atractiva puesto que esquiva el requisito — quizá inverosímil- de que la gente sepa algo de macroeconomía. Entonces, la cuestión es cómo forma estas percepciones el ciudadano medio, y la respuesta no es concluyente. De lo que sabemos hasta el momento, parece que la cobertura mediática de la economía objetiva podría ser un elemento importante a la hora de moldear las percepciones económicas de la gente. Por lo tanto, podría pensarse en un modelo en dos pasos en el cual la popularidad gubernamental dependiese de las percepciones populares de la economía y, a su vez, éstas dependiesen de la macroeconomía objetiva - aunque con una buena dosis de reinterpretación mediática que atenuaría el vínculo-. Además del papel de los medios de comunicación, otros agentes intermediarios con redes de información específicas — como sindicatos, partidos, líderes de opinióntambién podrían jugar un papel similar. 
¿Egotrópico o sociotrópico?

Éste es probablemente el debate más prominente entre los que las funciones de popularidad han engendrado. En la sección 1 ya hemos expuesto lo que se ha venido en llamar la hipótesis de responsabilidad: los votantes castigan a los gobiernos por las malas épocas económicas y los premian por las buenas. Este proceso podría generarse concebiblemente a partir de dos fundamentos psicológicos distintos.

De una parte, podría ser que los votantes tuvieran en consideración sus propias condiciones económicas para definir qué constituye una mala (o buena) época económica. En ese caso, los agravios económicos personales constituirían el estímulo para negar el apoyo al gobierno del momento (y viceversa). Dado que las condiciones macroeconómicas tan sólo son la agregación de los agravios y las venturas económicas personales, un deterioro económico tan sólo encarnaría la existencia de más gente experimentando agravios económicos personales $y$, por tanto, negando su apoyo al gobierno (y viceversa). El tipo de apoyo que es otorgado y negado a partir de este tipo de criterios egoístas es generalmente llamado apoyo egotrópico — también aludido como egocéntrico o pocketbook (de cartera).

De otra parte, también puede ser que los votantes tomen en consideración las condiciones económicas colectivas del país para definir qué constituyen épocas económicamente malas (o buenas). Aquí, las condiciones macroeconómicas no son tan sólo la agregación de agravios y venturas económicas personales, sino que son las propias condiciones macroeconómicas las que constituyen el estímulo para otorgar o negar el apoyo al gobierno del momento. Esto es lo que (desde Kinder y Kiewiet, 1979) se llama apoyo sociotrópico.

Es importante darse cuenta de que tanto el apoyo egotrópico como el sociotrópico son de naturaleza igualmente económica: tan sólo difieren en qué unidad económica priorizan. No obstante, tan sólo algunos investigadores negarían que el apoyo egotrópico es de una naturaleza más egoísta que el apoyo sociotrópico: éstos argüirían que el apoyo sociotrópico resultaría igualmente egoísta en caso que los individuos valoraran las condiciones económicas generales tan sólo como una aproximación verosímil de sus condiciones económicas personales. También deberíamos subrayar que tanto el apoyo egotrópico como el apoyo sociotrópico resultan igualmente compatibles con la hipótesis de responsabilidad y con las relaciones agregadas que se han descubierto entre condiciones macroeconómicas y apoyo al gobierno.

Uno de los puntos importantes a clarificar sobre el apoyo egotrópico se refiere a la unidad económica que el ciudadano se fija para percibir sus agravios y venturas económicas personales. En principio, la definición sugiere que la unidad debería ser tan sólo él/ella mismo/a, pero la literatura acepta que el hogar familiar es también una unidad económica en la que fijarse que es perfectamente válida bajo la hipótesis egotrópica. Pero una pregunta se mantiene: si podemos extender el concepto de agravios y venturas económicas 
personales al hogar familiar, ¿por qué no deberíamos extenderlo a círculos más amplios, como los parientes más lejanos, clanes, etc.? Que nosotros sepamos, la literatura no ha considerado todavía esta cuestión, pero una respuesta plausible podría ser que la mayoría de hogares familiares tienen una caja conjunta.

Una de las ventajas teóricas del apoyo egotrópico es que también esquiva el exigente requisito de que la gente se entere de macroeconomía: la gente respondería a la macroeconomía no porque se enteren de las tasas de inflación o de los datos de paro (sabemos que no se enteran), sino porque ellos y su entorno inmediato experimentan de hecho las consecuencias de la inflación y del paro. Algunos autores se han destacado defendiendo la existencia de resultados de tipo egotrópico (ej., Nannestad y Paldam, 1993a, 1993b).

La presunción egotrópica constituyó el consenso nunca manifestado explícitamente hasta que Kinder y Kiewiet publicaron sus clásicos artículos en 1979 y 1981. En esos artículos, Kinder y Kiewiet verbalizaron la presunción egotrópica, la concibieron como hipótesis y mostraron que:

- el descontento económico personal raramente influye sobre el voto;

- las medidas económicas personales no son estadísticamente significativas para explicar el voto al Congreso, así como sus cambios;

- ninguna medida económica personal influye sobre el voto en las elecciones presidenciales, excepto el paro experimentado personalmente;

- ninguna de esas medidas actúa tampoco indirectamente, a través de generar cambios en la identificación de partido;

... y por tanto rechazaron la hipótesis, concluyendo que las condiciones económicas personales no tienen consecuencias electorales. Como alternativa, los autores concibieron la hipótesis sociotrópica, la sometieron a pruebas equivalentes y mostraron que:

- la percepción de las condiciones económicas colectivas habitualmente influye sobre el voto;

- los juicios sobre la economía nacional son estadísticamente significativos para explicar el voto al Congreso y en las presidenciales;

- la percepción de las condiciones económicas colectivas mantienen su significación cuando se controlan por identificación de partido, así como también influyen sobre la intensidad de la identificación con el Partido Demócrata, de forma que muestran una influencia sobre el voto tanto directa como indirecta;

- la percepción de las condiciones económicas colectivas no son extrapolaciones de las medidas económicas personales, y ofrecen mejores resultados que éstas cuando se introducen conjuntamente en el modelo;

... y por tanto la conclusión inevitable fue que: 
"it is not how voters are affected by economic issues in a tangible, personal way that counts politically. The political consequences of economic conditions are not carried by personally experienced hardships. Rather, a citizen's political response to economic conditions is mediated by judgements that are collectively oriented - perceptions, for example, that business conditions in the country have turned sour, that the unemployment rate is climbing, that inflation is worsening nationwide" (...) «in reaching political decisions, voters draw not on their recent personal economic experiences, but on information about aggregate economic conditions" (p. 499).

A partir de los artículos cardinales de Kinder y Kiewiet, la mayoría de autores han apoyado la hipótesis sociotrópica, que eventualmente ha devenido la posición ortodoxa en este debate. De hecho, los sucesivos sondeos de la literatura de Paldam informan básicamente de resultados sociotrópicos (Paldam, 1981; Nannestad y Paldam, 1994; véase también Lewis-Beck, 1988).

Uno de los principales problemas para la hipótesis sociotrópica es que parece excesivamente exigente para el ciudadano medio: ¿cómo puede responder electoralmente ante las condiciones económicas del país - y no ante las personales- si sabemos que el ciudadano medio no se entera de mucho en lo que se refiere a estas condiciones? Una respuesta es que no sería necesario que los ciudadanos supieran mucho de macroeconomía para responder a las condiciones económicas - de hecho, sólo deberían formarse percepciones muy toscas-. A su vez, esas percepciones podrían ser explicadas por cobertura mediática, líderes de opinión, etc., como ya se anticipó en el epígrafe anterior (Economía objetiva o...).

No obstante, la mente de los críticos se ha empañado inmediatamente por una suspicacia: ¿qué pasaría si esas percepciones fueran meras racionalizaciones de una simpatía preexistente para con el partido gobernante? Entonces, la popularidad del gobierno sería "explicada" por una variable que es, a su vez, explicada por la simpatía para con el partido gobernante - y la totalidad de la función de popularidad devendría poco más que una elegante tautología-. Esa posibilidad será discutida más adelante. Volvamos al debate que nos ocupaba.

Se han divisado muchos procedimientos para resolver el debate y establecer si la relación es fundamentalmente egotrópica o sociotrópica. Quizás el más directo -y por tanto el más comprensible- requiere incluir en el modelo algunas preguntas de encuesta sobre la percepción tanto de las condiciones económicas personales como de las condiciones económicas nacionales. Entonces, la cuestión es básicamente una cuestión empírica: cuál de ellas obtiene una mayor significación estadística.

Nannestad y Paldam (1994, pp. 225-226) reseñan otro procedimiento más ingenioso. Básicamente arguye que parece realista que cada persona que experimenta el paro en carne propia pueda provocar un cambio de voto en tan sólo un número limitado de votantes (pongamos tres). Por lo tanto, si el coeficiente que el modelo estima para la variable paro fuera menor que 0,03 , ello indicaría 
que el impacto del paro sobre la popularidad del gobierno es factible que actuara a través del paro personalmente experimentado por el interesado o por alguna persona próxima. No obstante, si el coeficiente que el modelo estima para la variable paro fuera mucho mayor que 0,03 , ello indicaría que la mayor parte del impacto del paro sobre la popularidad del gobierno no actúa a través del paro personalmente experimentado por el interesado o por alguna persona próxima, sino que actúa a través de algunas referencias colectivas sobre el paro. Dado que el coeficiente habitual para la variable paro suele tener un valor alrededor de 0,6, ello refutaría inevitablemente la hipótesis egotrópica.

Habiendo encontrado que algunas funciones nacionales de popularidad aparecen como egotrópicas y algunas emergen como sociotrópicas, los académicos han intentado explicar la disparidad en términos de la hipótesis cultural: en los países - como Dinamarca- donde se suele hacer responsable al Estado del bienestar económico de los ciudadanos, los votantes tenderán a evaluar el gobierno del momento de acuerdo con la eficacia con que cumplan esa función, mostrando así un voto egotrópico; pero en aquellos países - como los EE.UU.- donde no se suele hacer responsable al Estado del bienestar económico de los ciudadanos individuales, sería absurdo que se evaluara el gobierno de acuerdo con esa función y, por tanto, los votantes tendrán mayor tendencia a evaluar el gobierno del momento de acuerdo con su gestión del bienestar económico de la nación entera, mostrando así un voto sociotrópico.

La hipótesis cultural es interesantísima desde un punto de vista epistemológico, puesto que introduce la cuestión de la aplicabilidad de un mismo modelo teórico a realidades políticas e institucionales muy distintas. En esos intentos de investigación comparada, es fundamental plantearse la cuestión de si explicamos casos específicos o pretendemos una explicación de validez universal. La aplicación de este modelo a España - donde los ciudadanos atribuyen al Estado una responsabilidad sobre su bienestar muy superior a los países anglosajones- será una experiencia fundamental para verificar la hipótesis cultural.

\section{¿Prospectivo o retrospectivo?}

En la sección 2 hemos mostrado que la componente económica del modelo suele contener dos tipos distintos de variables, ambas referidas a la percepción de la economía: las expectativas económicas (es decir, percepción sobre el futuro de la economía) y las restrospecciones económicas (es decir, percepción sobre el pasado de la economía). Una cuestión académicamente polémica es cuáles son más eficaces a la hora de influir la popularidad gubernamental, si las expectativas económicas o las retrospecciones económicas.

De hecho, Downs ya perfiló un esbozo en que las expectativas eran los datos racionales que debían tenerse en cuenta para decidir el voto. Es más, las retrospecciones tan sólo merecían cierto crédito en caso que pudieran ser utili- 
zadas para formar las expectativas relevantes sobre el futuro. No obstante, la investigación empírica sobre funciones de popularidad parecen apoyar la idea de que los votantes dejan que las retrospecciones económicas influyan sus votos. El promotor de ese punto de vista es Fiorina $(1978,1981)$, pero el grueso de la academia le ha apoyado (ver, por ejemplo, el compendio de LewisBeck, 1988; Nannestad y Paldam, 1993a). A pesar de ello, algunos autores han apoyado también el bando de las expectativas (Chappel y Keech, 1985; Sanders et al., 1987).

\section{¿Cuán miopes resultamos ser?}

La componente de estructura aludida en la sección 2 ha mostrado una variable muy importante que aparece en la gran mayoría de funciones de popularidad: la tasa de descuento. Tal como se dijo allí, esa variable recoge cuánta popularidad gubernamental del período anterior se mantiene en el período presente. Así, pues, es una muy buena medida de la permanencia o inercia de la popularidad pasada.

Por la misma razón, la tasa de descuento también es una medida indirecta de la influencia continuada de las condiciones económicas o políticas que ya ejercieron su influencia puntual en el período anterior. Mostrémoslo: en primer lugar, esas condiciones pasadas influyeron la popularidad que el gobierno logró durante el período pasado; en segundo lugar, una parte de esa popularidad se mantiene en el período presente; y, por tanto, en tercer lugar, aquellas condiciones todavía influyen en parte la popularidad gubernamental del presente. Imaginemos que la Navidad genera un jovial aumento de la popularidad gubernamental: debido a la tasa de descuento, el gobierno todavía retendrá una parte de esa popularidad navideña en Año Nuevo; de hecho, una pequeña parte de la popularidad gubernamental generada por unas Navidades especialmente joviales todavía se mantendrá en Semana Santa —aunque aquel espíritu navideño haga ya mucho tiempo que se haya esfumado.

Tasas de descuento bajas producen un desfallecimiento muy rápido de los efectos producidos por los acontecimientos pasados: por ejemplo, una tasa de descuento del 0,6 implicaría que más del 90 por 100 de cualquier breve soplo de popularidad gubernamental estaría ya consumido pasados cinco períodos $\left(0,6^{5}\right)$. Contrariamente, altas tasas de descuento indican una repercusión muy duradera de los acontecimientos pasados sobre la popularidad gubernamental: por ejemplo, una tasa de descuento de 0,87 indicaría que más o menos la mitad de cualquier aumento momentáneo de la popularidad gubernamental todavía se mantendría en pie una vez pasados cinco períodos de la desaparición del acontecimiento causante $\left(0,87^{5}\right)$. En resumidas cuentas, esta alta tasa de descuento señalaría el hecho de que la gente evalúa el gobierno teniendo en cuenta acontecimientos que ya hace mucho tiempo que pasaron - pero que todavía pueden recordar-. Esto es lo que se llama hipermetropia del votante. 
Al contrario, la previa baja tasa de descuento nos indicaría que - a la hora de evaluar al gobierno- la gente olvida rápidamente sus logros y sus deslices y, por tanto, lo que cuenta más es la situación actual. Esto se llama miopía del votante, a veces aludido como cortedad de miras.

La mayoría de hallazgos empíricos sugieren que los votantes son tremendamente miopes. Tan sólo Hibbs y Vasilatos (1981) y Hibbs (1982) encontraron signos de hipermetropía -aunque sus resultados han sido muy debatidos y algunos los juzgan cuestionables.

Es interesante darse cuenta de que es plausible que la hipótesis egotrópica produjera individuos hipermétropes, puesto que los agravios personales son más difíciles de olvidar que las frías condiciones macroeconómicas. También se ha dado el caso de encontrar mayores signos de miopía en las funciones de popularidad que en las funciones de voto. Ello es probablemente debido al hecho que las encuestas de popularidad son situaciones gratuitas que son propicias para generar respuestas impulsivas y emocionales por parte de los encuestados. Parece razonable que esas respuestas se basarían fundamentalmente en emociones primarias sobre las condiciones circunstanciales del momento. En vez de ello, el sufragio real se produce después de una campaña y suele ser un acto mucho más responsable y premeditado. Como consecuencia de ello, es más probable que el sufragio se base en evaluaciones a largo plazo. Nannestad y Paldam (1994, p. 229) caricaturizan graciosamente esta situación diciendo que cuando uno está insatisfecho con el gobierno, no es lo mismo echar una pataleta (situación de encuesta) que echarlos a patadas (situación de sufragio). $\mathrm{O}$ tal como lo dicen ellos: it is rather different kicking out the incumbents than just kicking them.

\section{La atribución de responsabilidad}

La sección 1 argumentó que las funciones de popularidad están basadas en la llamada hipótesis de responsabilidad: es decir, que los gobiernos son castigados por el mal rendimiento económico y premiados por el bueno. El mismo nombre de la hipótesis sugiere que el gobierno es visto como el agente responsable de los resultados macroeconómicos. Pero no siempre pasa así. De hecho, muchas veces nos las vemos con administraciones que no son responsables de la gestión macroeconómica - o al menos no son contempladas como tales.

Uno de esos casos son los gobiernos minoritarios en sistemas pluripartidistas. Esos gobiernos suelen disfrutar de muy poco margen de maniobra y a menudo ejercen muy poco control sobre la macroeconomía. En esas circunstancias, parecería razonable que el ciudadano no atribuyera demasiada responsabilidad económica al partido en el gobierno. Una situación similar ocurre en gobiernos de coalición, puesto que sería casi imposible que el votante medio pudiera atribuir a cada uno de los partidos coaligados una parte alícuota de responsabilidad por los resultados económicos. En esos casos, la hipótesis de 
responsabilidad suele desmoronarse y las funciones de popularidad devienen no significativas. Resulta que la mayoría de Estados democráticos entran en una de estas categorías, pero la atención académica habitual sobre EE.UU., Gran Bretaña y Alemania (tres países con gobiernos insólitamente mayoritarios y estables) ha hecho olvidar que el mundo no siempre funciona así, ; ni siquiera frecuentemente!

Otro caso de atribución de responsabilidad problemática aparece en ejecutivos duales, donde la gestión económica es compartida (o al menos lo parece). En esos casos, la investigación empírica ha mostrado que la popularidad presidencial suele seguir mucho más de cerca a las condiciones económicas que no la popularidad del partido mayoritario. En ese caso, una mayor visibilidad del presidente propiciaría una mayor atribución de responsabilidad (incluso con cierta independencia del reparto constitucional de competencias).

Además, algunas administraciones simplemente no gestionan la política macroeconómica. Ése es el caso de los gobiernos locales y regionales, y también el de la Unión Europea. Dadas sus actividades más habituales, no hay razón por la cual a esas instituciones se les debieran atribuir responsabilidades por los resultados macroeconómicos. Incluso a nivel empírico, algunos estudios han discutido que la popularidad de esas instituciones pudiera ser modelada a través de las circunstancias económicas (Bosch y Newton, 1995). En esas instituciones, un fracaso de sus funciones de popularidad -y el consiguiente rechazo de la hipótesis de responsabilidad- apuntarían probablemente al hecho de no ser sujetos de la atribución de responsabilidad.

\section{¿Explicamos algo?}

La explicación científica es una cuestión espinosa: no importa cuánto nos esforcemos en aportar relaciones consistentes, siempre quedará la posibilidad de que esa relación sea espúrea, o tautológica, o una mera racionalización... o muchas otras amenazas metodológicas igualmente inquietantes.

En las funciones de popularidad, la principal amenaza es la siguiente: aun cuando verifiquemos que las expectativas económicas muestran una influencia muy clara sobre la popularidad gubernamental, no podemos estar muy seguros de que no haya una simpatía progubernamental preexistente que produzca ambas actitudes (popularidad gubernamental y expectativas económicas), revelando así una relación meramente espúrea. Existe una posibilidad incluso peor: la gente para la que el gobierno se ha hecho más popular racionaliza con posterioridad esa popularidad incrementando sus percepciones macroeconómicas, por lo que la relación es en realidad una mera racionalización y no una explicación causal.

Por si esto no fuera ya bastante, esos peligros son incluso alentados por una forma de medir las percepciones económicas ciertamente extravagante que Lewis-Beck (1988) ha venido en llamar preguntas complejas: en ellas a los indi- 
viduos no se les pregunta si sus economías han mejorado o empeorado, sino si las politicas gubernamentales han hecho que sus economías mejorasen o empeorasen. Parece claro que, con estas preguntas, el peligro de que las percepciones económicas sean una mera racionalización de una simpatía progubernamental preexistente resulta incrementado. Si esas preguntas llegasen a producir un mejor ajuste en el modelo, eso no revelaría la consecución de una explicación más eficaz, sino que indicaría que nos estamos aproximando más a una relación claramente tautológica.

Estos peligros no se pueden descartar completamente, pero algunos autores han utilizado trucos ingeniosos para evitarlos en alguna medida (desde luego, otros autores han fracasado en el mismo empeño). Primero, se ha comprobado estadísticamente que la popularidad gubernamental sigue a las percepciones económicas (y no las precede). Segundo, se han introducido controles adecuados (por ejemplo, preferencia de partido) para eludir relaciones espúreas. Tercero, una mayoría de autores han usado variables independientes que son lo suficientemente distintas de la popularidad gubernamental como para evitar tautologías, explicaciones circulares y meras racionalizaciones. Cuarto, con objeto de asegurarse de que las variables independientes económicas no son la consecuencia de la popularidad gubernamental sino su antecedente genuino, algunos autores incluso han intentado un paso más y modelar estas percepciones económicas como una función de la economía real. Quinto, se ha dirigido mucha atención a la plausibilidad de la relación y no sólo a la significación estadística del modelo; aunque es admitido que se requiere una observancia mucho más rigurosa de esta cuestión. Finalmente, todavía existe un salvamento in extremis del valor explicativo de las funciones de popularidad: su capacidad predictiva. A eso nos dedicamos en el siguiente epígrafe.

\section{¿Predecimos correctamente?}

Aunque la predicción siempre estuvo en la mente de los expertos en funciones de popularidad y de sus lectores, sólo recientemente algunos expertos han acometido la arriesgada tarea de predecir la popularidad gubernamental futura usando un modelo de series temporales estimado con anterioridad. Sanders (1991) es un ejemplo exitoso de ello, puesto que predijo la victoria conservadora de 1992 con un grado de exactitud impresionante... ¡dos años antes de las elecciones!

Desde luego, la corrección de las predicciones implicaría un mayor crédito para la solidez de una función de popularidad, puesto que apuntaría al hecho de que no se trata de un mero artefacto estadístico, sino que describe una historia real que subyace en la evolución de la popularidad gubernamental. En alguna medida, unas predicciones correctas serían una confirmación de la capacidad explicativa de la función de popularidad. Cabe destacar que un positivismo radical argumentaría que las predicciones correctas son la confirmación 
definitiva de la capacidad explicativa de una función de popularidad, pero a nosotros esto nos parece una exageración.

No obstante, el hecho de que alguna vez una función haya predicho con éxito la popularidad gubernamental no es una garantía absoluta de nada: la mayoría de acontecimientos momentáneos no pueden ser anticipados y, en la medida en que esos acontecimientos desvían la popularidad de su pauta regular, la capacidad predictiva de la función resultaría fuertemente afectada. Por lo tanto, una función exitosa se puede desmoronar en cualquier momento debido a la aparición de un acontecimiento inesperado, por lo que nunca sabemos si la función predecirá correctamente la próxima vez. Ésta es la amenaza que siempre cuelga sobre su capacidad predictiva. Si la amenaza se materializa y uno debe predecir en presencia de un acontecimiento inesperado, la única esperanza para la función de popularidad reside en la existencia (por otra parte bastante habitual) de una baja tasa de descuento: esa tasa implicaría que el impacto del acontecimiento inesperado sobre la popularidad gubernamental se extinguiría con prontitud, y el modelo retomaría su capacidad predictiva rápidamente.

Curiosamente, la exactitud de las predicciones anteriores también puede ser el origen de futuras inexactitudes: la misma difusión del modelo podría alertar a los votantes de las manipulaciones a las cuales están siendo sometidos $y$, por lo tanto, podría introducir cambios en el comportamiento de los ciudadanos. Éste sería un caso de predicción autodesmentida. De hecho, nosotros no creemos que la función sea una oportunidad para que los gobiernos manipulen a los electores (sabemos que lo intentan aun sin esa función), sino una oportunidad para que los ciudadanos conozcan el riesgo y se protejan ante él.

\section{LOS ESTUDIOS RECIENTES}

Por último, vamos a finalizar en esta última sección con un breve repaso a las aportaciones más recientes a la literatura sobre funciones de popularidad y voto, haciendo hincapié en los resultados obtenidos, que están relacionados con los debates a los que hemos hecho referencia anteriormente.

Tal como hemos dicho al principio, últimamente se han realizado diversos intentos de generalización de las funciones de popularidad. Entre ellos, cabe citar los estudios de Mattila (1996) y de Midtbø (1998), que estiman ambos una única función para el conjunto de los países escandinavos; el de Anderson y Junishii (1997), que, en el caso del Japón, amplían el estudio de las funciones de voto a los partidos que no están en el gobierno, y el de Pacek y Radcliff (1995), que aplican las teorías del voto económico a un conjunto de países en vías de desarrollo en los que ha habido una serie ininterrumpida de elecciones competitivas y de las que se dispone de datos (Botswana, Costa Rica, India, Jamaica, Sri Lanka, Trinidad y Tobago, Uruguay y Venezuela). Las conclusiones de estos estudios confirman que la hipótesis de la responsabilidad no fun- 
ciona para gobiernos minoritarios o de coalición (como es el caso de los países nórdicos), que en los países en vías de desarrollo existe una asimetría en el efecto de la economía en el voto (una recesión tiene enormes costes electorales para los gobiernos pero el crecimiento económico no supone ningún beneficio) y el efecto es mayor que en los países desarrollados, y que la apertura comercial, que afecta enormemente a determinadas economías como la japonesa, es una variable clave en la modelización de la popularidad del gobierno.

Otra característica de los estudios recientes es un constante esfuerzo para mejorar la metodología empleada, así como la aplicación de técnicas cada vez más sofisticadas. Entre ellos cabe destacar el de Nannestad y Paldam (1997), donde los autores analizan los posibles sesgos de los modelos, tanto de los dinámicos como de los estáticos (sobre los cuales ya había alertado Kramer en su famoso artículo de 1983 "The Ecological Fallacy Revisited»), y realizan un diseño de investigación que intenta compensarlos. Además, utilizan datos de un macro pseudopanel construido con 28 encuestas trimestrales a la población danesa. Mattila (1996) utiliza este mismo tipo de datos, conocidos como pooled cross-national data, pero combinando los de distintos países en vez de los de distintas encuestas, aunando así las ventajas de los modelos dinámicos con las de los estáticos. Los artículos de Clarke, Rapkin y Stewart (1994), de Clarke y Stewart (1995 y 1996) y de Clarke, Stewart y Whiteley (1997) destacan por la utilización de Error Correction Models, modelos que capturan la tendencia a largo plazo (habitualmente perdida cuando se toman diferencias de variables no estacionarias), y por la aplicación de numerosos tests que permiten comparar modelos alternativos.

En los estudios recientes parece existir un cierto consenso sobre la importancia de las variables económicas subjetivas en los modelos de popularidad. Nadie pone en duda su significación y alta capacidad explicativa. El énfasis actual se pone en analizar el origen de las mismas, en establecer su correlación con otras variables objetivas y subjetivas y en decidir cuál de ellas es mejor predictor de la popularidad gubernamental. George C. Edwards III, William Mitchell y Reed Welch (1995) investigan en su artículo la formación de las percepciones de la gente. De sus resultados se desprende que la visibilidad de los temas económicos y de política exterior, magnificada principalmente por los medios de comunicación, mediatiza el impacto que dichos temas tienen en las funciones de popularidad de los gobernantes. Por su parte, Clarke, Rapkin y Stewart (1994) Ilegan a la conclusión que las variables económicas objetivas y subjetivas tienen efectos independientes en la popularidad. Clarke y Stewart (1995) intentan determinar cuál de las cuatro variables subjetivas (expectativas personales, expectativas colectivas, evaluación retrospectiva personal y evaluación retrospectiva colectiva) funciona mejor en el caso británico. Estiman cuatro modelos alternativos, uno con cada una de ellas, llegando a la conclusión que ninguno de ellos es superior a los demás. Cuando se incluye también la evaluación del Primer Ministro como variable explicativa, los modelos con percepciones sobre la economía personal son mejores que los construidos con las 
percepciones sobre la situación general, pero que no hay evidencia para establecer la superioridad de las expectativas económicas sobre la evaluación retrospectiva, o viceversa. Los mismos autores, Clarke y Stewart (1996), con datos canadienses, llegan a la conclusión que la variable que afecta directamente la popularidad es la sociotrópica (percepción sobre la situación económica general), pero que la variable egotrópica (percepción sobre la evolución de la situación económica personal) actúa indirectamente a través de su influencia en la formación de las percepciones sobre la economía nacional. El último de los artículos de este grupo, el de Clarke, Stewart y Whiteley (1997), establece que la evaluación del Primer Ministro y la identificación partidista son variables explicativas de la popularidad, que mantienen su significación cuando se controla por variables económicas subjetivas y por variables políticas. Los autores establecen un modelo en dos etapas, donde la evaluación del Primer Ministro y la identificación partidista se modelizan a partir de variables subjetivas, variables macroeconómicas y variables políticas. La comparación con modelos alternativos muestra que el modelo estimado es claramente superior a los demás. Las conclusiones del artículo de Nannestad y Paldam (1997) insisten en la misma dirección de los anteriores de estos mismos autores: el electorado danés es egotrópico. Finalmente, Diana C. Muntz y Jeffery J. Mondak (1997) inciden en la polémica entre egotrópico-sociotrópico, mostrando que la gente tiene unas percepciones sobre la situación económica de distintos grupos sociales que son distintas e independientes de las percepciones sobre la situación individual y de las percepciones sobre la situación general. De forma sorprendente para los propios autores, sus resultados apuntan a la existencia de un efecto de «justicia sociotrópica» en la popularidad presidencial. Es decir, la popularidad del Presidente aumenta si los votantes tienen la percepción que los cambios económicos han afectado de igual manera a todas las clases sociales.

Un último artículo que parece interesante citar es el de Petrocik (1996), que aporta datos que respaldan la hipótesis del policy-oriented voting, es decir, del voto en función de la reputación del partido como competente para manejar determinados problemas (generalmente el paro, los partidos de izquierda, y la inflación, los de derecha).

\section{REFERENCIAS}

AMOr Bravo, Elías M. (1985): «El ciclo político de los negocios y su referencia al caso español (1976-1985)», Información Comercial Española, núm. 2015, del 13 al 29 de diciembre.

ANDERSON, Christopher J., y JunishII (1997): "The Political Economy of Election Outcomes in Japan", British Journal of Political Science, 27: 619-659.

Bellucci, P. (1991): «Italian Economic Voting: A Deviant Case or Making Case for Better Theory», en H. Norpoth, M. S. Lewis-Beck y J.-D. Lafay (eds.), Economics and Politics: The Calculus of Support, Ann Arbor, Michigan University Press (pp. 63-84).

Bosch, A., y NEwTon, K. (1995): "Economic Calculus or Familiarity Breeds Content", en O. Niedermayer y R. Sinnot (eds.), Public Opinion and Internationalized Governance, Oxford, Oxford University Press. 
Clarke, H. D., y Steward, M. (1995): «Economic Evaluations, Prime Ministerial Approval and Governing Party Support: Rival Models Reconsidered", British Journal of Political Science, 25: $145-170$.

- (1996): «Economists and Electorates: The Subjective Economy of Governing party Support in Canada", European Journal of Political Science, 29: 191-214.

Clarke, H. D.; Rapkin, J., y STeward, M. (1994): “A President Out of Work: A Note on the Political Economy of presidential Approval in the Bush Years", British Journal of Political Science, 24: 535-561.

Clarke, H. D.; Steward, M., y Zuk, G. (1986): «Politics, Economics and Party Popularity in Britain, 1979-1983n, Electoral Studies, 5: 123-141.

Clarke, H. D.; Steward, M., y Whiteley, P. (1997): «Tory trends: Party Identification and the Dynamics of Conservative Support Since 1992", British Journal of Political Science, 27: 299-319.

Clarke, H. D.; Mishler, W., y Whiteley, P. (1990): «Recapturing the Falklands: Models of Conservative Popularity, 1979-1983", British Journal of Political Science, 20: 63-81.

Chappeli, H. W., Jr., y Keech, W. R. (1985): "A New View of Political Accountability for Economic Performance», American Political Science Review, 79 (1): 10-27.

Downs, Anthony (1957): An Economic Theory of Democracy, New York, Harper y Row (Trad. castellana: Teoría económica de la Democracia, Madrid, Aguilar, 1973).

EDwards III, George C.; MrTCHell, William, y WeLCH, Reed (1995): «Explaining Presidential Approval: The Significance of Issue Salience», American Journal of Political Science, 39 (1): 108-134.

FIORINA, M. P. (1978): «Economic Retrospective Voting in American national Elections: A Micro-Analysis", American Journal of Political Science, 22: 426-443.

- (1981): Retrospective Voting in American National Elections, New Haven, Yale University Press.

Frey, B. S., y SCHNeIder, F. (1978): "A Politico-Economic Model of the United Kingdom", Economic Journal, 88: 243-253.

- (1979): "An Econometric Model with an Endogenous Government Sector", Public Choice, 34: 29-43.

Golden, David G., y Poterba, James M. (1980): "The Price of Popularity: The Political Business Cycle Reexamined", American Journal of Political Science, 24: 696-714.

Goonhart, C. A. E., y Bhansali, R. J. (1970): "Political Economy», Political Studies, 18: 43 106.

HibBs, D. A., Jr. (1979): «The Mass Public and Macroeconomic Performance: The Dynamics of Public Opinion Toward Unemployment and inflation", American Journal of Political Science, 23: 705-731.

- (1982): "On the Demand for Economic Outcomes: Macroeconomic Outcomes and Mass Political Support in the United States, Great Britain and Germany", Journal of Politics, 44: 426-462.

Hibis, D. A., Jr., y Vasilatos, N. (1981): “Economics and Politics in France: Economic Performance and Mass Political Support for Presidents Pompidou and Giscard d'Estaing", European Journal of Political Research, 9: 133-145.

INOGUCHI, T. (1980): "Economic Conditions and Mass Support in Japan 1960-1976», en P. Whiteley (ed.), Models of Political Economy, London y Beverly Hills, Sage, pp. 121-154.

JONUNG, Lars, y WADENSJO, Eskil (1987): "Rational, Adaptative and Learning Behavior of Voters: Evidence from Disagregated Popularity Functions for Sweden", Public Choice, 54: 197-210.

KIEWEIT, Roderick D. (1981): "Policy-Oriented Voting in Response to Economic Issues", American Political Science Review, 75: 448-459.

KINDER, Donald R., y KIEWEIT, Roderick D. (1981): "Sociotropic Politics: The American Case», British Journal of Political Science, II (2): 129-161.

Kramer, Gerald H. (1971): «Short-term Fluctuations in U.S. Voting Behavior, 1896-1964», American Political Science Review, 65: 131-143. 
Krampr, Gerald H. (1983): "The ecological Fallacy Revisited: Aggregate- versus Individual-level Findings on Economics and Elections, and Sociotropic Voting", American Political Science Review, 7 (1): 92-111.

LEWIS-BFCK, M. S. (1988): Economics and Elections: The Major Western Democracies, Ann Arbor, Michigan University Press.

Mancha Navarro, Tomás (1993): Economia y votos en España, Madrid, Instituto de Estudios Económicos.

Matтlia, Mikko (1996): "Economic Changes and Government Popularity in Scandinavian Countries", British Journal of Political Science, 26: 583-595.

MintBu, Tor (1998): "The Open Policotomy: A Ddynamic Analysis of Social democratic popularity and economic Policies in Scandinavia", British Journal of Political Science, 28: 93-112.

Mulillek, John E. (1970): "Presidential Popularity from Truman to Johnson", American Political Science Review, 64: 18-34.

MUNT/, Diana C., y MONDAK, Jeffrey J. (1997): "Dimensions of Sociotropic behavior: GroupBased Judgements of Fairness and Well-being", American Journal of Political Science, 41 (1): 284-308.

Nannesial), Peter, y Paidda, Martin (1993a): "Mellem Samfundsøkonomi og egeninteresse: Samfundsøkonomi, privatøkonomi og mistillid til regeringens kompetence», økonnomy of Politik. (English version as Working Paper: «Economic Grievances and Government Support in Small Welfare State: Micro Theory and the danish casem.)

- (1993b): «Sammenhægen mellem samfundsøkonomi, privatøkonomi, og tilslutningen tl regeringspartierne», Politica, 25: 189-206. (English version as Working Paper: "The Egotropic Welfare Man: A Micro Study of Danish Economic Voting, 1985-92, using the Marcus Model».)

- (1994): "The VP-function: A Survey of the Literature on Vote and Popularity Functions after 25 Years", Public Choice, 79: 213-245.

- (1997): "From the Pocketbook of the Welfare Man: A Pooled Cross-Section Study of Economic Voting in Denmark, 1986-92", British Joumal of Political Science, 27: 119-137.

Norinhaus, William D. (1975): "The Political Business Cycle", Review of Economic Studies, 42 : 169-190.

Norjol1, Helmut (1987): "The Falklands War and Government Popularity", Electoral Studies, 6: 3-16.

Pacik, Alexander, y Ranchir, Benjamin (1995): "The Political Economy of Competitive Elections in the Developing World", American Journal of Political Science, 39 (3): 745-759.

Pald)AM, Martin (1981): "A Preliminary Survey of the Theories and Findings on Vote and Popularity Functions", European Journal of Political Research, 9: 181-199.

- (1986): "The Distribution of Election Results and the Two Explanations of the Cost of Ruling", European Journal of Political Economy, 2 (1): 5-24.

PAImAM, M., y SchnFII)R, F. (1980): "The Macro-Economic Aspects of Government and Opposition Popularity in Denmark, 1957-1978", Nationalokonomist Tidsskrift, 2: 149-170.

PETROCIK, John R. (1996): "Issue Ownership in Presidential Elections, with a 1980 Case Study", American Journal of Political Science, 40 (3): 825-850.

RATIINGik, H. (1991): "Unemployment and Elections in West Germany", en H. Norpoth, M. S. Lewis-Beck y J.-D. Lafay (eds.), Economics and Politics: The Calculus of Support, Ann Arbor, Michigan University Press (pp. 49-62).

SAN1Frs, David (1991): "Government Popularity and the Next General Election", Political Quarterly, 62: 235-261.

SANiders, David; WAR1), Hugh, y MArsh, David (1987): "Government Popularity and the Falklands War: A Reassessment", British Journal of Political Science, 17 (3): 281-313.

Schumplik, Joseph (1945): Capitalism, Socialism and Democracy, New York, Harper.

TuF'l'E, Edward R. (1978): Political control of the Economy, Princeton, Princeton University Press. 


\begin{abstract}
This paper reviews the research conducted on the relationship between the evolution of the voting intention and the economic situation. It notes that citizens tend to reward governments which rule during periods of prosperity by voting for them and vice-versa. This paper examines the way these functions have been shaped: the types of variables they encompass, the temporal delays/time lags used and their explicatory value. It concludes by entering the principle debates which have been engendered by the functions of popularity: Is the vote more influenced by the evolution of the voter's economic conditions than by the evolution of the country's macro-economic conditions? Do appraisals of the past carry more weight than expectations of the future? In another paper which is about to be published, the authors will present their findings regarding a function of popularity for the Spanish government between 1984 and 1996.
\end{abstract}

